\title{
RACETRACK SHAPED MUON STORAGE RINGS FOR A NEUTRINO FACTORY *
}

\author{
C. Johnstone ${ }^{\dagger}$, FNAL, Batavia, IL 60510, USA \\ A. Garren, UCLA, Los Angeles, USA
}

\begin{abstract}
20-GeV and 50-GeV lattices have been designed for muon storage rings at Brookhaven National Laboratory (BNL) and Fermi National Accelerator Laboratory (FNAL), respectively. Only recent work on the $20-\mathrm{GeV}$ ring will be discussed in detail here as the $50-\mathrm{GeV}$ ring has been well documented[1]. The 20-GeV designs presented in this paper supplement the lattice developed at BNL for the Feasibility II Study of a Neutrino Factory[2]. The principal difference between these designs is the use of an unconventional type of magnet in the Feasibility II Study design and of conventional magnets in the ones presented here.
\end{abstract}

\section{INTRODUCTION}

A planer racetrack geometry was chosen because it maximizes the production efficiency for neutrinos directed to a single detector site. (However, if it is necessary to preserve polarization and to service detectors at two distant sites, the 'bowtie' geometry is required.) With a racetrack layout, the entire ring is tilted with the production straight directed downward towards the neutrino detector. Site constraints limit the vertical drop of the ring at the FNAL site and the BNL site to 600' and $<40$, respectively. The tilt angle and the allowed vertical drop determine the length of the major axis of the ring, and, therefore, the relative length of the production straight. In the $50-\mathrm{GeV}$ ring design, which assumes a neutrino source at Fermilab and a detector on the SLAC site, about $40 \%$ of the muons that decay produce neutrinos in the production straight. The $20-\mathrm{GeV}$ ring at the Brookhaven Laboratory site, is directed to a detector located in Carlsbad, NM. In order to keep this ring above the water table, the length of the ring, and, likewise, that of the production straight, is limited, so the production efficiency is lower than that of the $50-\mathrm{GeV}$ ring. In order to maximize its efficiency the arcs were made very compact, which motivated the initial adoption of an arc magnet design with skew-quadrupole focusing. As an alternative, the two designs discussed in this paper use conventional magnets, where the magnetic fields have mid- plane symmetry. One design uses combined-function magnets in the arcs, the other one uses separated-function magnets. The combined-function lattice is partly based on that of the $50 \mathrm{GeV}$ Fermilab lattice (although the arcs of that design are separated function).

\footnotetext{
* Work supported by the Universities Research Association, Inc., under contract DE-AC02-76CH00300 with the U.S. Department of Energy. ‘cjj@fnal.gov
}

\section{GENERAL}

The racetrack design is simple and economical; it contains a downward straight, called the production straight, a return straight pointed towards the surface, and two arcs, together with dispersion suppressors and matching sections. The rings have periodicity one, but have reflection symmetry about the short axis.

The two lattices described in this paper are symmetric, in contrast to the antisymmetric lattice designed at BNL. We now proceed to a more detailed discussion of the two $20 \mathrm{GeV}$ conventional-magnet lattices.

\section{ARCS}

\subsection{Combined Function Ring Arc}
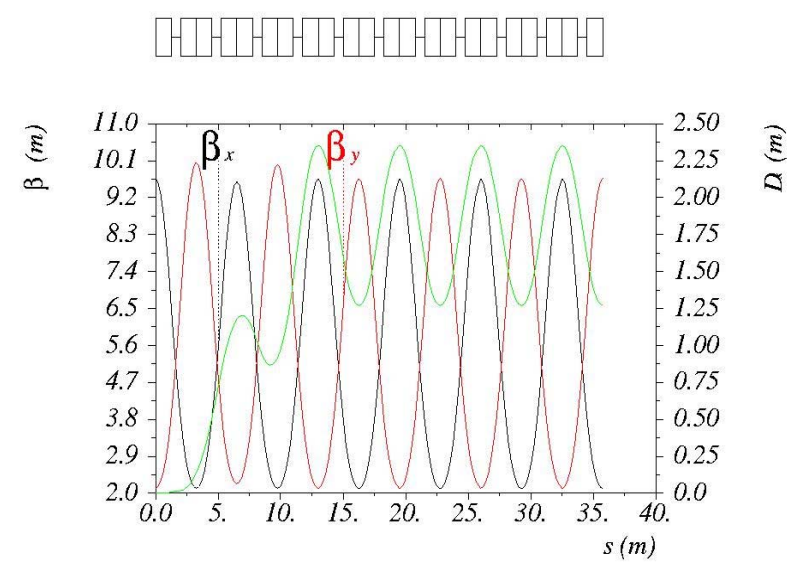

Figure 1 Combined Function Ring Arc:

$$
\beta x \text { - black, } \beta y \text { - red, Dx - green }
$$

The lattice of a full arc is shown in Figure 1. Both of the long straights have zero dispersion. The dispersion in the arcs is suppressed differently on the ends next to the production straight and those next to the return straight. On the return straight side, the two end arc cells have dipoles with half the normal bend angle. On the production straight side, the end cells are normal arc ones and the long matching drift is used to suppress dispersion by correctly positioning a normal-bend (0-gradient) dipole. In Figure 2, the left-hand side of the graph shows the return straight dispersion suppressor and the righthand side the normal arc end before the combined matching and dispersion suppression section. The parameters of the arc cells are given in Table 1 together with those of the separated-function arc design discussed in the following section. 


\subsection{Separated Function Ring Arc}

The separated-function arc lattice, composed of sectorshaped, zero-gradient dipoles, is shown in Figure 2 with parameters listed in Table 1.

The dispersion is brought to zero at the ends of each arc by reducing both the length and the bending angle of the two cells at each end by an amount such that the product of length and angle is equal to half that of the central cells of the arc. This method, which was applied by one of the authors for the SSC collider lattice, is designed to suppress the dispersion at the arc ends without significantly reducing the dipole packing efficiency.

To improve the quality of the neutrino beam, a change of the above bending pattern has been included in the design. Specifically, high-divergence neutrinos generated in the upstream matching region of the production straight are diverted from reaching the detector by a bump magnet placed at each end of the production region. Then, in order to preserve zero dispersion in this region, it is necessary to shorten the $5^{\text {th }}$ and $6^{\text {th }}$ dipoles from the left of Figure $2,(2 \pi$ in phase from the bump magnet).

Table 1. Parameters of the arc cells

\begin{tabular}{|l|l|c|c|}
\hline & Unit & $\begin{array}{c}\text { Comb. } \\
\text { functi } \\
\text { on }\end{array}$ & $\begin{array}{c}\text { Separate } \\
\text { function }\end{array}$ \\
\hline ARC CELLS & & & \\
\hline Number per arc & & 3.5 & 3 \\
\hline Cell length & $\mathrm{m}$ & 6.5 & 9.69 \\
\hline Guide Field & $\mathrm{T}$ & 7.3 & 7.3 \\
\hline Intermagnet spacing & $\mathrm{m}$ & 0.75 & 0.7 \\
\hline Dipoles & & & \\
\hline Length & $\mathrm{m}$ & 2.5 & 2.73 \\
\hline Strength (k1) & $\mathrm{m}^{-2}$ & 0.024 & 0 \\
\hline Quadrupoles & & & \\
\hline Length QF / QD & $\mathrm{m}$ & - & $0.70 / 0.77$ \\
\hline Strength & $\mathrm{m}^{-2}$ & - & 0.4 \\
\hline DISP. SUP. CELLS & & & \\
\hline Number per arc & & 2 & 4 \\
\hline Dipoles & & & \\
\hline Length & $\mathrm{m}$ & 2.5 & 1.69 \\
\hline Strength (k1) & $\mathrm{m}^{-2}$ & 0.024 & 0 \\
\hline Quadrupoles & & & \\
\hline Length QF / QD & $\mathrm{m}$ & - & $0.97 / 1.01$ \\
\hline Strength & $\mathrm{m}^{-2}$ & - & 0.4 \\
\hline
\end{tabular}

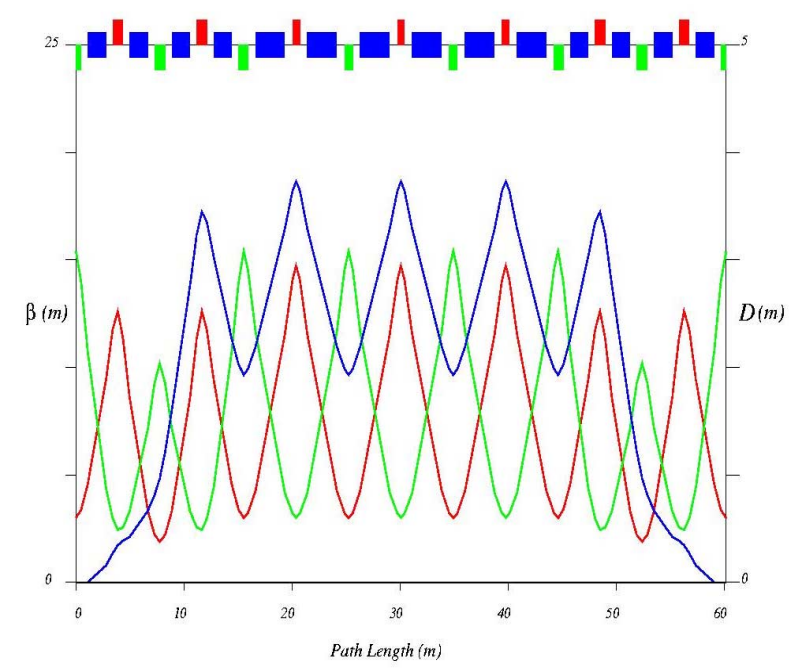

Figure 2 Separated Function Ring Arc $\beta \mathrm{x}$ - red, $\beta \mathrm{y}$ - green, Dx - blue

\section{PRODUCTION STRAIGHT SECTION}

\subsection{Combined Function Ring Production Straight}

The straight section, shown in Figure 3, contains the high beta neutrino-production region and two matching regions with parameters described in Table 2.

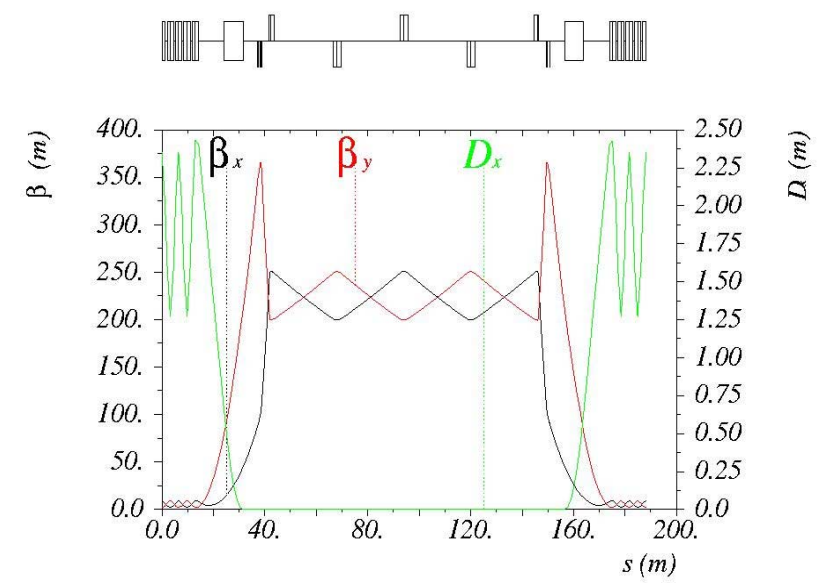

Figure 3 Combined Function, Production Side Half

Ring: $\beta \mathrm{x}$ - black, $\beta \mathrm{y}-\mathrm{red}, \mathrm{Dx}-$ green

\subsection{Separated Function Ring Production Straight}

In the separated-function design, the production region (Figure 4) is magnet free, and the matching sections each contain five quadrupoles. Parameters are given in Table2. 


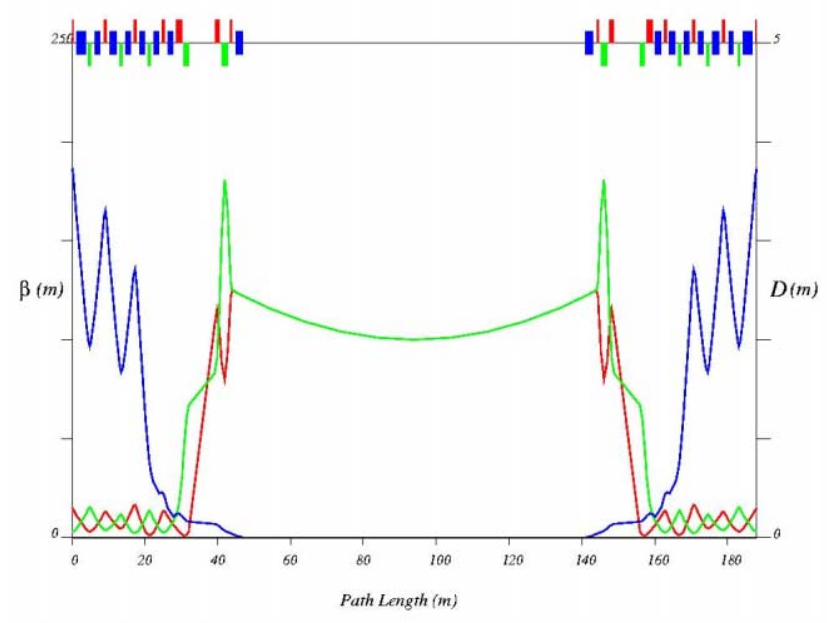

Figure 4. Separated Function Production Side Half Ring: $\beta \mathrm{x}-$ red, $\beta \mathrm{y}$ - green, Dx - blue

\section{RETURN STRAIGHT SECTION}

\subsection{Combined Function Ring Return Straight}

The return straight shown in Figure 5, contains $2090^{\circ}$ cells. The parameters are shown in Table 2 . The return straight section is used for beam injection and extraction, the rf system and phase adjustment. It is made up of periodic cells with quadrupoles and drifts.

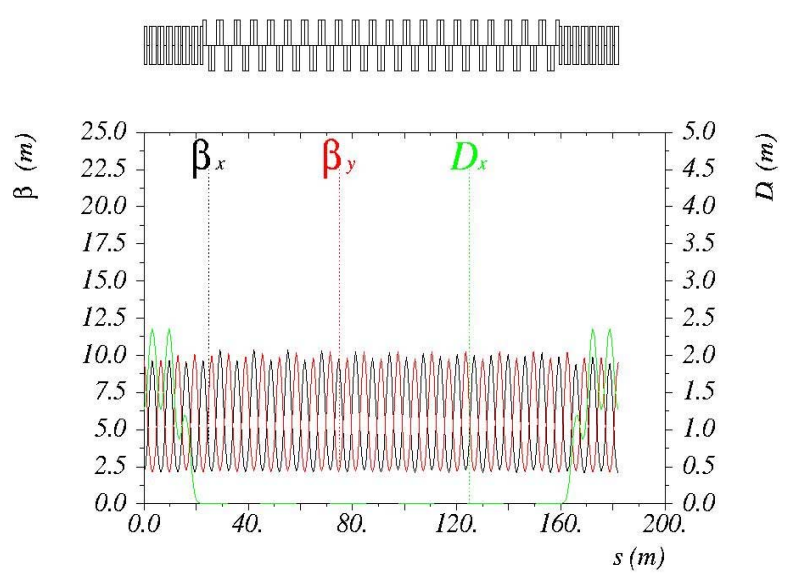

Figure 5 Separated Function Return Side Half Ring $\beta \mathrm{x}$ - black, $\beta \mathrm{y}$ - red, Dx - green

\subsection{Separated Function Ring Return Straight}

Here, the return straight contains $1490^{\circ}$ cells, 9.14-m long. The parameters of these cells are shown in Table 5, and the lattice functions in Figure 4. Two cells at each end are used for matching from the arc cells, three cells on each side of the center of the straight for injection/extraction, and four cells in the center of the straight for a phase trombone.

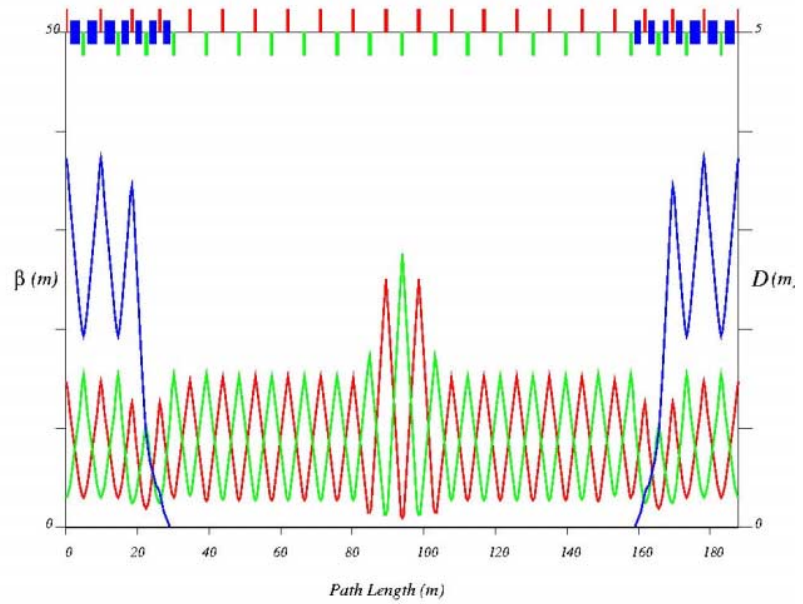

Figure 6. Separated Function Return Side Half Ring $\beta \mathrm{x}$ - red, $\beta \mathrm{y}$ - green, Dx - blue

Table 2. Parameters of the $20-\mathrm{GeV}$ Storage Rings

\begin{tabular}{|c|c|c|c|}
\hline & Unit & $\begin{array}{l}\text { Comb. } \\
\text { Function }\end{array}$ & $\begin{array}{l}\text { Separate } \\
\text { Function }\end{array}$ \\
\hline \multicolumn{4}{|l|}{ GENERAL } \\
\hline Circumference & $\mathrm{m}$ & 377 & 376 \\
\hline Neut. decay fract. & $\%$ & 28 & 25 \\
\hline Length / width & $\mathrm{m}$ & $166 / 38$ & $166 / 38$ \\
\hline Declination & & $13^{\circ}$ & $13^{\circ}$ \\
\hline Vertical drop & $\mathrm{m}$ & 38 & 37 \\
\hline Normalized emit. $\bullet_{n}$ & $\begin{array}{l}\mathrm{m}- \\
\mathrm{rad}\end{array}$ & 0.03 & 0.03 \\
\hline $\mathrm{dp} / \mathrm{p}(\mathrm{rms})$ & $\%$ & 1.6 & 1.6 \\
\hline$\cdot x(\max ) / \cdot y(\max )$ & & $250 / 366$ & $125 / 181$ \\
\hline $\mathrm{vx} \quad /$ & & $6.34 /$ & $8.18 /$ \\
\hline vy & & 6.30 & 7.28 \\
\hline $\mathrm{vx} \cdot / \mathrm{vy^{ \bullet }}$ & & $-8 /-8$ & $-12 /-12$ \\
\hline \multicolumn{4}{|l|}{ PROD. INSERT } \\
\hline Match region length & $\mathrm{m}$ & 32 & 17 \\
\hline Prod. region length & $\mathrm{m}$ & 104 & 94 \\
\hline$-\cdot(\max )$ & $\mathrm{m}$ & 250 & 125 \\
\hline$-\bullet$ & $\mathrm{m}^{-1}$ & 0.01 & 0.01 \\
\hline Beam Divergence & $\mathrm{mr}$ & 0.5 & 0.5 \\
\hline \multicolumn{4}{|l|}{ RETURN INSERT } \\
\hline$\cdot x(\max ) / \cdot y(\max )$ & $\mathrm{m}$ & $10 / 10$ & $25 / 28$ \\
\hline
\end{tabular}

\section{REFERENCES}

[1] N. Holtkamp and D. Finley, eds., "A Feasibility Study of a Neutrino Source Based on a Muon Storage Ring," Fermilab-Pbu-00/108-E (2000).

[2] S. Ozaki, R. Palmer, M. Zisman, and J. Gallardo, "Feasibility Study II of a Muon-Based Neutrino Source", BNL-52623, June 2001. 